\title{
5D Extremal Rotating Black Holes and CFT duals
}

\author{
Farhang Loran!1, Hesam Soltanpanah:2 \\ Department of Physics, Isfahan University of Technology, \\ Isfahan 84156-83111, Iran
}

\begin{abstract}
Kerr/CFT correspondence has been recently applied to various types of 5D extremal rotating black holes. A common feature of all such examples is the existence of two chiral CFT duals corresponding to the U(1) symmetries of the near horizon geometry. In this paper, by studying the moduli space of the near horizon metric of five dimensional extremal black holes which are asymptotically flat or AdS, we realize an $\mathrm{SL}(2, \mathbb{Z})$ modular group which is a symmetry of the near horizon geometry. We show that there is a lattice of chiral CFT duals corresponding to the moduli points identified under the action of the modular group. The microscopic entropy corresponding to all such CFTs are equivalent and are in agreement with the Bekenstein-Hawking entropy.
\end{abstract}

\footnotetext{
${ }^{1}$ e-mail: loran@cc.iut.ac.ir

${ }^{2}$ e-mail: h_soltanpanahi@ph.iut.ac.ir
} 


\section{Contents}

1 Introduction

2 Extremal 5D Rotating Black Holes

3 Asymptotic Symmetry Group 3

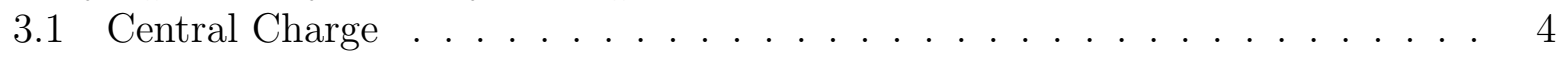

4 Frolov-Thorne Temperature and Entropy

5 Consistency of boundary conditions 6

\section{Introduction}

The statistical mechanical interpretation of the Bekenstein-Hawking entropy of black holes seems to be an everlasting source of inspiration in quantum gravity. The work of Strominger and Vafa [1] which is known as the first attempt to calculate entropy of black holes by counting the corresponding microstates implies that, in principle any gravity solution should have various CFT duals.

A recent approach in this direction is the Kerr/CFT correspondence [2], which is intrinsically a generalization of Brown-Henneaux approach [3, 4] to AdS/CFT correspondence [5, 6, 7]. Extremal Kerr-AdS metrics in 5, 6 and 7 dimensions are studied in [8], and BMPV black hole is studied in [9]. CFT dual of 5D extremal rotating Kaluza-Klein black holes is investigated in [10]. Some examples of extremal black hole/CFT correspondence in 4D and 5D gauged and ungauged supergravities are considered in [11. Embedding of such correspondence in string theory for some 5D solutions is studied in [12, 13. All of these calculations are based on the periodicity of the circles of the near horizon metric and enhancement of the corresponding $U(1)$ symmetries to the Virasoro of a dual chiral CFT. By choosing special boundary conditions, it is shown that there is a CFT dual associated to each of the circles, with the same CFT entropy that is in agreement with the Bekenstein-Hawking entropy.

In this paper we study the moduli space of the near horizon metric of five dimensional extremal rotating black holes that are asymptotically flat or AdS. We show that there is an $\mathrm{SL}(2, \mathbb{Z})$ modular group generated by the usual transformations $\tau \rightarrow \tau+1$ and $\tau \rightarrow$ $-1 / \tau$, where $\tau$ is a definite moduli of the geometry, that leaves the Bekenstein-Hawking entropy invariant. The operation $\tau \rightarrow-1 / \tau$ interchanges the two circles of the near horizon geometry, so maps the CFT's corresponding to these circles into each other. The operator $\tau \rightarrow \tau+1$ leaves one of the circles intact but adds it once to the other circle. These operations are symmetries of the near horizon geometry but map a given CFT dual to another CFT. Thus the $\mathrm{SL}(2, \mathbb{Z})$ modular group assigns a lattice of CFT duals corresponding to the moduli points identified under the action of the modular group. By using the Cardy formula we show that the CFT entropy of all of these CFTs are equivalent and are in precise agreement with the Bekenstein-Hawking entropy.

The organization of this paper is as follows. In section 92 , we study the moduli space of the near horizon geometry of 5D extremal rotating black holes that are asymptotically flat or AdS. In section $\$ 3$ we use the Brown-Henneaux method to define the Virasoro generators 
in terms of the asymptotic symmetry group of near horizon geometry and compute the associated central charge. In section $\$ 4$ by studying the behavior of the matter field near the horizon of the black hole we compute the Frolov-Thorne (FT) temperature [17] and the CFT entropy for the lattice of the CFT duals. The consistency of boundary conditions is shown in section 95 .

\section{Extremal 5D Rotating Black Holes}

In the Brown-Henneaux method [3, 4], the first step to construct the CFT dual to a geometry, is to realize the metric of the near horizon geometry [2, ㅇ, 9, 10, 11, 12, 13]. The general form of near horizon of rotating black holes in 5D that are asymptotically flat or AdS is given in [14]. In this paper we follow the conventions of [11,

$$
d s^{2}=-\left(1+\hat{r}^{2} l^{-2}\right) d \hat{t}^{2}+\frac{d \hat{r}^{2}}{1+\hat{r}^{2} l^{-2}}+\hat{r}^{2}\left(d \theta^{2}+\cos ^{2} \theta d \hat{\phi}_{1}^{2}+\sin ^{2} \theta d \hat{\phi}_{2}^{2}\right),
$$

where $l^{-2}$ is the cosmological constant. For deriving the near horizon geometry of the extremal limit, it is suitable to use the following coordinate transformations [1]

$$
\hat{r}=r_{0}(1+\varepsilon r), \quad \hat{\phi}_{1}=\phi_{1}+\Omega_{1}^{0} \hat{t}, \quad \hat{\phi}_{2}=\phi_{2}+\Omega_{2}^{0} \hat{t}, \quad \hat{t}=\frac{t}{2 \pi r_{0} T_{H}^{\prime 0} \varepsilon},
$$

where $r_{0}$ is the radius of horizon in the extremal limit, $\Omega_{i}^{0}$ are the angular velocities on the horizon, and the quantity $T_{H}^{\prime}$ is derivative of Hawking temperature with respect to the outer horizon radius at itself,

$$
T_{H}^{\prime 0}:=\left.\frac{\partial T_{H}}{\partial r_{+}}\right|_{r_{+}=r_{0}} .
$$

The near horizon metric of the extremal five dimensional black hole is obtained in the $\varepsilon \rightarrow 0$, which gives

$$
\begin{aligned}
& d s^{2}=A(\theta)\left(-r^{2} d t^{2}+\frac{d r^{2}}{r^{2}}\right)+F(\theta) d \theta^{2}+B_{1}(\theta)\left(e_{1}+C(\theta) e_{2}\right)^{2}+B_{2}(\theta) e_{2}^{2}, \\
& e_{1}=d \phi_{1}+k_{1} r d t, \\
& e_{2}=d \phi_{2}+k_{2} r d t,
\end{aligned}
$$

where $A, B_{i}, C$ and $F$ are only functions of $\theta, 0 \leq \phi_{i} \leq 2 \pi$ and $0 \leq \theta \leq \pi$. This metric can be viewed as an $\mathrm{S}^{3}$ bundle over $\mathrm{AdS}_{2}$. The constants $k_{i}=\left(2 \pi T_{i}\right)^{-1}$, in which $T_{i}$ are the FT temperatures defined as follows [11],

$$
T_{i}=\lim _{r_{+} \rightarrow r_{0}} \frac{T_{H}}{\Omega_{i}^{0}-\Omega_{i}}=-\frac{T_{H}^{\prime 0}}{\Omega_{i}^{\prime 0}} .
$$

Thus the parameter space of asymptotically flat or AdS 5D black holes, is seven dimensional with parameters $A, B_{i}, C, F, k_{i}$.

The last two terms of the metric (41) can be realized as a torus

$$
d s_{\mathbb{T}^{2}}^{2}=\left(R_{1}\right)^{2}\left|e_{1}+\tau e_{2}\right|^{2}
$$

where

$$
R_{1}=\sqrt{B_{1}}, \quad \tau=C+i \sqrt{\frac{B_{2}}{B_{1}}} .
$$


The generators of the $\mathrm{SL}(2, \mathbb{Z})$ modular group of $\mathbb{T}^{2}$ are

$$
\tau \rightarrow-\frac{1}{\tau}, \quad \tau \rightarrow \tau+1
$$

The action of the modular group of the torus can be extended to the parameter space of the near horizon geometry (4) as follows

$$
\begin{aligned}
& \left(C, B_{1}, B_{2}, k_{1}, k_{2}\right) \rightarrow\left(-C|\tau|^{-2}, B_{1}|\tau|^{2}, B_{2}|\tau|^{-2},-k_{2}, k_{1}\right), \\
& \left(C, B_{1}, B_{2}, k_{1}, k_{2}\right) \rightarrow\left(C+1, B_{1}, B_{2}, k_{1}-k_{2}, k_{2}\right),
\end{aligned}
$$

with $A$ and $F$ invariant. The corresponding change in the metric (44) can be compensated by a redefinition of the $\phi_{i}$ coordinates given by

$$
\begin{array}{ll}
\phi_{1} \rightarrow \tilde{\phi}_{1}=-\phi_{2}, & \phi_{2} \rightarrow \tilde{\phi}_{2}=\phi_{1}, \\
\phi_{1} \rightarrow \tilde{\phi}_{1}=\phi_{1}-\phi_{2}, & \phi_{2} \rightarrow \tilde{\phi}_{2}=\phi_{2} .
\end{array}
$$

Thus, the SL $(2, \mathbb{Z})$ modular transformations (9) and (10) are symmetries of the near horizon geometry (4) 3 Consequently, the Bekenstein-Hawking entropy for the extremal black hole

$$
S_{B H}=\frac{1}{4} \int d \theta d \phi_{1} d \phi_{2} \sqrt{F(\theta) B_{1}(\theta) B_{2}(\theta)}
$$

is invariant under the $\operatorname{SL}(2, \mathbb{Z})$ modular group. Here and henceforth we set $G_{5}=1$.

\section{$3 \quad$ Asymptotic Symmetry Group}

The asymptotic symmetry group (ASG) of a near horizon metric is the group of allowed symmetries modulo trivial symmetries. By definition, an allowed symmetry transformation obeys the specified boundary conditions [2]. A possible boundary condition for the fluctuations around the geometry (41) is,

$$
h_{\mu \nu} \sim \mathcal{O}\left(\begin{array}{ccccc}
r^{2} & 1 / r^{2} & 1 / r & r & r \\
& 1 / r^{3} & 1 / r^{2} & 1 / r & 1 / r \\
& & 1 / r & 1 / r & 1 / r \\
& & & 1 & 1 \\
& & & & 1
\end{array}\right)
$$

in the basis $\left(t, r, \theta, \phi_{1}, \phi_{2}\right)$. This boundary conditions are consistent with the $\operatorname{SL}(2, \mathbb{Z})$ symmetry of the near horizon geometry given in (4). This should be contrasted against the

\footnotetext{
${ }^{3}$ By transformations (11) and (12), the orientation and the periods of the near horizon geometry do not change.
} 
boundary conditions,

$$
\begin{aligned}
& h_{\mu \nu}^{(1)} \sim \mathcal{O}\left(\begin{array}{ccccc}
r^{2} & 1 / r^{2} & 1 / r & 1 & r \\
& 1 / r^{3} & 1 / r^{2} & 1 / r & 1 / r^{2} \\
& & 1 / r & 1 / r & 1 / r \\
& & & 1 & 1 \\
& & & & 1 / r
\end{array}\right), \\
& h_{\mu \nu}^{(2)} \sim \mathcal{O}\left(\begin{array}{ccccc}
r^{2} & 1 / r^{2} & 1 / r & r & 1 \\
& 1 / r^{3} & 1 / r^{2} & 1 / r^{2} & 1 / r \\
& & 1 / r & 1 / r & 1 / r \\
& & & 1 / r & 1 \\
& & & & 1
\end{array}\right),
\end{aligned}
$$

considered in [8, 9, 10, 11, 12, 13] which are not consistent with the modular transformation $\tau \rightarrow \tau+1$ and by $\tau \rightarrow-1 / \tau$ get mapped into each other.

It is easy to show that the general diffeomorphism preserving the boundary conditions (14) is given by,

$$
\begin{aligned}
\zeta & =\left[C+\mathcal{O}\left(\frac{1}{r^{3}}\right)\right] \partial_{t}+\left[r \epsilon\left(\phi_{1}, \phi_{2}\right)+\mathcal{O}(1)\right] \partial_{r}+\mathcal{O}\left(\frac{1}{r}\right) \partial_{\theta} \\
& +\left[\lambda_{1}\left(\phi_{1}, \phi_{2}\right)+\mathcal{O}\left(\frac{1}{r^{2}}\right)\right] \partial_{\phi_{1}}+\left[\lambda_{2}\left(\phi_{1}, \phi_{2}\right)+\mathcal{O}\left(\frac{1}{r^{2}}\right)\right] \partial_{\phi_{2}}
\end{aligned}
$$

where $\epsilon\left(\phi_{1}, \phi_{2}\right)$ and $\lambda_{i}\left(\phi_{1}, \phi_{2}\right)$ are arbitrary smooth periodic functions of $\phi_{1}$ and $\phi_{2}$. The traceless condition for $h_{\mu \nu}$ indicates that

$$
\epsilon\left(\phi_{1}, \phi_{2}\right)+\partial_{\phi_{1}} \lambda_{1}\left(\phi_{1}, \phi_{2}\right)+\partial_{\phi_{2}} \lambda_{2}\left(\phi_{1}, \phi_{2}\right)=0
$$

Thus the ASG contains a class of generators

$$
\zeta=\lambda_{1}\left(\phi_{1}, \phi_{2}\right) \partial_{\phi_{1}}+\lambda_{2}\left(\phi_{1}, \phi_{2}\right) \partial_{\phi_{2}}-r\left[\partial_{\phi_{1}} \lambda_{1}\left(\phi_{1}, \phi_{2}\right)+\partial_{\phi_{2}} \lambda_{2}\left(\phi_{1}, \phi_{2}\right)\right] \partial_{r}
$$

A subalgebra of the generators (19) is a Virasoro algebra $\left[\zeta_{m}, \zeta_{n}\right]_{\text {Lie }}=-i(m-n) \zeta_{m+n}$, where

$$
\zeta_{m}=-e^{-i m \phi_{1}} \partial_{\phi_{1}}-e^{-i m \phi_{2}} \partial_{\phi_{2}}-i m r\left(e^{-i m \phi_{1}}+e^{-i m \phi_{2}}\right) \partial_{r}
$$

corresponding to $\lambda_{i}\left(\phi_{1}, \phi_{2}\right)=\lambda_{i}\left(\phi_{i}\right), i=1,2$ in Eq.(19) $)$.

\subsection{Central Charge}

Charges associated to the diffeomorphisms (17) are defined by [15, 16],

$$
Q_{\zeta}=\frac{1}{8 \pi} \int_{\partial \Sigma} k_{\zeta}[h, g],
$$

where $\partial \Sigma$ is spatial surface at infinity and

$$
\begin{aligned}
k_{\zeta}[h, g] & =\frac{1}{2}\left[\zeta_{\nu} \nabla_{\mu} h-\zeta_{\nu} \nabla_{\sigma} h_{\mu}^{\sigma}+\zeta_{\sigma} \nabla_{\nu} h_{\mu}^{\sigma}+\frac{h}{2} \nabla_{\nu} \zeta_{\mu}\right. \\
& \left.-h_{\nu}^{\sigma} \nabla_{\sigma} \zeta_{\mu}+\frac{1}{2} h_{\nu \sigma}\left(\nabla_{\mu} \zeta^{\sigma}+\nabla^{\sigma} \zeta_{\mu}\right)\right] *\left(d x^{\mu} \wedge d x^{\nu}\right),
\end{aligned}
$$


in which $*$ denotes the Hodge dual in 5D. Since we are interested in the solution which remains extremal we set $Q_{\partial_{t}}$ to zero.

In the Brown-Henneaux approach [3] the central charge is given by

$$
\frac{1}{8 \pi} \int_{\partial \Sigma} k_{\zeta_{m}}\left[\mathcal{L}_{\zeta_{n}}, g\right]=-\frac{i}{12} c\left(m^{3}-m\right) \delta_{m+n, 0} .
$$

For the metric (40) and diffeomorphisms (20), the central charge is

$$
c=\frac{3\left(k_{1}+k_{2}\right)}{2 \pi} \int d \theta d \phi_{1} d \phi_{2} \sqrt{F(\theta) B_{1}(\theta) B_{2}(\theta)}=\frac{6\left(k_{1}+k_{2}\right) S_{B H}}{\pi} .
$$

That is, $c=c_{1}+c_{2}$ where $c_{i}=6 \pi^{-1} k_{i} S_{B H}, i=1,2$, are the central charges of the two CFTs associated to the $\phi_{i}$ circles in [11. This result is in agreement with the central charge $c=c_{\text {grav }}+c_{\text {gauge }}$ assigned to the four-dimensional Kerr-Newmann-AdS-dS black hole viewed as a $5 \mathrm{D}$ solution [18].

In general, the central charge of each point in the lattice of CFT duals defined by Eqs.(9) and (10) is given by

$$
c_{(m, n)}=\frac{6\left(m k_{1}+n k_{2}\right) S_{B H}}{\pi},
$$

where $m=a+c$ and $n=b+d$ in which,

$$
\left(\begin{array}{ll}
a & b \\
c & d
\end{array}\right) \in S L(2, \mathbb{Z}) .
$$

Notice that, the $\mathrm{SL}(2, \mathbb{Z})$ modular symmetry is absent if one of the $k_{i}$ is zero (like supersymmetric 5D black ring) since the components $g_{t \phi_{i}}$ are not of the same order in $r$. For such cases the central charge in the Brown-Henneaux approach is expected to be equivalent to the central charge that can be calculated in the usual AdS/CFT approaches. This is verified for the supersymmetric 5D black ring in [19].

\section{Frolov-Thorne Temperature and Entropy}

The FT temperature can be determined by identifying quantum numbers of a matter field in the near horizon geometry with those in original geometry [8]. For the chiral CFT given by (20) a matter field can be expanded in eigen modes of the asymptotic energy $\omega$ and angular momentum $m$ as

$$
\Phi=\sum_{\omega, m, l} \varphi_{\omega m l} e^{-i\left(\omega t-m \phi_{+}\right)} f_{l}(r, \theta), \quad \phi_{ \pm}=\phi_{1} \pm \phi_{2}
$$

since $\left[\zeta_{0}, \phi_{-}\right]=0$. Using (2), the identification

$$
e^{-i \omega \hat{t}+i m \hat{\phi}_{+}}=e^{-i n_{t} t+i n \phi_{+}}
$$

implies that

$$
n=m, \quad \omega=2 \pi r_{0} T_{H}^{\prime 0} n_{t}+\left(\Omega_{1}^{0}+\Omega_{2}^{0}\right) n .
$$

Considering the Boltzmann factor,

$$
e^{-\frac{\omega-m\left(\Omega_{1}+\Omega_{2}\right)}{T_{H}}}=e^{-\left(\frac{n_{t}}{T_{t}}+\frac{n}{T}\right)}
$$


one obtains

$$
\frac{1}{T_{t}}=\frac{2 \pi r_{0} T_{H}^{\prime 0}}{T_{H}} \quad \frac{1}{T}=\frac{\left(\Omega_{1}^{0}-\Omega_{1}\right)+\left(\Omega_{2}^{0}-\Omega_{2}\right)}{T_{H}} .
$$

It is easy to show that at the extremal limit we have

$$
T_{t}=0, \quad \frac{1}{T}=\frac{1}{T_{1}}+\frac{1}{T_{2}},
$$

where $T_{i}=\left(2 \pi k_{i}\right)^{-1}$ are the FT temperatures of the two CFTs which are associated to the $\phi_{i}$ circles in [11]. The FT temperature of each point in the lattice of CFT duals is given by

$$
T_{(m, n)}=\frac{1}{2 \pi\left(m k_{1}+n k_{2}\right)}
$$

where $m$ and $n$ are similar to the ones used in Eq.(25). Using the Cardy formula, one can obtain the statistical entropy of dual CFT as follows which is in precise agreement with the Bekenstein-Hawking entropy (13),

$$
S_{\text {mic }}=\frac{\pi^{2}}{3} c_{(m, n)} T_{(m, n)}=S_{\mathrm{BH}}
$$

\section{Consistency of boundary conditions}

In this section we give explicitly the form of charges associated to the time translation generator $\partial_{t}$ and the general diffeomorphism generators given in Eq.(19). As mentioned in Eq.(21) the charge associated to the generator $\zeta$ is given by

$$
Q_{\zeta}=\frac{1}{8 \pi} \int_{\partial \Sigma} k_{\zeta}[h, g]
$$

in which

$$
\begin{aligned}
k_{\zeta}[h, g] & =\frac{1}{2}\left[\zeta_{\nu} \nabla_{\mu} h-\zeta_{\nu} \nabla_{\sigma} h_{\mu}{ }^{\sigma}+\zeta_{\sigma} \nabla_{\nu} h_{\mu}{ }^{\sigma}+\frac{h}{2} \nabla_{\nu} \zeta_{\mu}\right. \\
& \left.-h_{\nu}{ }^{\sigma} \nabla_{\sigma} \zeta_{\mu}+\frac{1}{2} h_{\nu \sigma}\left(\nabla_{\mu} \zeta^{\sigma}+\nabla^{\sigma} \zeta_{\mu}\right)\right] *\left(d x^{\mu} \wedge d x^{\nu}\right)
\end{aligned}
$$

Given $k_{\partial_{t}}$ in Eq.(37), one verifies that the generator of time translation $Q_{\partial_{t}}=0$. This is a necessary condition for consistency of our calculations. We had started with extremal solutions (4) and $Q_{\partial_{t}}=0$ ensures that by the boundary conditions (14) extremality will not be violated. Furthermore, since the component of $k_{\zeta}$ given in Eq.(39), that contribute in $Q_{\zeta}$ is independent of $r$, the charges $Q_{\zeta}$ are finite under the boundary condition (14). 
The explicit form of $k_{\partial_{t}}$ is given by 4

$$
\begin{aligned}
k_{\partial_{t}} & =\frac{r}{A^{2} B_{1} B_{2}} d\left[\left(10 B_{1} B_{2}^{2} C k_{1} k_{2}-6 A B_{1} B_{2}\right)\left(\lambda_{1} d \phi_{2}+\lambda_{2} d \phi_{1}\right)\right. \\
& +\left(A^{2} B_{1}+A^{2} B_{2} C-A B_{1} B_{2} k_{2}^{2}\right)\left(\frac{\partial^{2} \lambda_{1}}{\partial \phi_{2}^{2}} d \phi_{2}+\frac{\partial^{2} \lambda_{2}}{\partial \phi_{2}^{2}} d \phi_{1}\right) \\
& +\left(A^{2} B_{2}-A B_{1} B_{2} k_{1}^{2}\right)\left(\frac{\partial^{2} \lambda_{1}}{\partial \phi_{1}^{2}} d \phi_{2}+\frac{\partial^{2} \lambda_{2}}{\partial \phi_{1}^{2}} d \phi_{1}\right) \\
& -2\left(A^{2} B_{2} C+A B_{1} B_{2} k_{1} k_{2}\right)\left(\frac{\partial^{2} \lambda_{1}}{\partial \phi_{1} \partial \phi_{2}} d \phi_{2}+\frac{\partial^{2} \lambda_{2}}{\partial \phi_{1} \partial \phi_{2}} d \phi_{1}\right) \\
& +4 B_{1} B_{2}\left(k_{2}{ }^{2} B_{2} \lambda_{1} d \phi_{2}+k_{1}{ }^{2} B_{1} \lambda_{2} d \phi_{1}\right) \\
& +6 B_{1} B_{2}\left(k_{1}{ }^{2} B_{1} \lambda_{1} d \phi_{2}+k_{2}{ }^{2} B_{2} \lambda_{2} d \phi_{1}\right) \\
& +2 B_{1} B_{2}{ }^{2} C\left(k_{1}{ }^{2} \lambda_{2} d \phi_{2}+k_{2}{ }^{2} \lambda_{1} d \phi_{1}\right)+2 B_{1} B_{2}{ }^{2} k_{1} k_{2}\left(\lambda_{2} d \phi_{2}+\lambda_{1} d \phi_{1}\right) \\
& \left.+2 B_{1} B_{2}{ }^{2} C^{2}\left(3 k_{1}{ }^{2} \lambda_{1} d \phi_{2}+2 k_{1}{ }^{2} \lambda_{2} d \phi_{1}+k_{1} k_{2} \lambda_{1} d \phi_{1}\right)\right]_{\partial \Sigma} \wedge d \theta+k_{\partial_{t}}^{\perp}
\end{aligned}
$$

where $k_{\partial_{t}}^{\perp}$ includes terms with components transverse to the boundary. So,

$$
\int_{\partial \Sigma} k_{\partial_{t}}^{\perp}=0
$$

Likewise,

$$
\begin{aligned}
k_{\zeta} & =\frac{1}{2 A^{2}}\left[2\left(\frac{\partial \lambda_{1}}{\partial \phi_{1}} \lambda_{2}-\lambda_{1} \frac{\partial \lambda_{2}}{\partial \phi_{1}}+\lambda_{1} \frac{\partial \lambda_{2}}{\partial \phi_{2}} C\right) B_{2}\left(k_{1} C+k_{2}\right)\right. \\
& -2 \frac{\partial \lambda_{1}}{\partial \phi_{2}} \lambda_{2}\left(k_{1} B_{1}+k_{2} B_{2} C\right)+k_{2} A \frac{\partial^{2} \lambda_{1}}{\partial \phi_{1}^{2}}\left(\frac{\partial \lambda_{2}}{\partial \phi_{2}}+\frac{\partial \lambda_{1}}{\partial \phi_{2}}\right) \\
& +2 A \lambda_{1}\left(k_{1} \frac{\partial^{3} \lambda_{2}}{\partial \phi_{1}^{2} \partial \phi_{2}}+k_{2} \frac{\partial^{3} \lambda_{1}}{\partial \phi_{1}^{2} \partial \phi_{2}}\right)+k_{1} A \frac{\partial \lambda_{1}}{\partial \phi_{1}}\left(\frac{\partial^{2} \lambda_{1}}{\partial \phi_{1}^{2}}+\frac{\partial^{2} \lambda_{2}}{\partial \phi_{1} \partial \phi_{2}}\right) \\
& +k_{2} A\left(\frac{\partial^{2} \lambda_{1}}{\partial \phi_{2}^{2}} \frac{\partial \lambda_{2}}{\partial \phi_{2}}+\frac{\partial \lambda_{1}}{\partial \phi_{2}} \frac{\partial^{2} \lambda_{2}}{\partial \phi_{1} \partial \phi_{2}}\right) \\
& +k_{1} A \frac{\partial \lambda_{2}}{\partial \phi_{1}}\left(\frac{\partial^{2} \lambda_{1}}{\partial \phi_{1} \partial \phi_{2}}+\frac{\partial^{2} \lambda_{2}}{\partial \phi_{2}^{2}}\right)+2 k_{1}\left(B_{1} \lambda_{1} \frac{\partial \lambda_{2}}{\partial \phi_{2}}-B_{2} C^{2} \lambda_{2} \frac{\partial \lambda_{1}}{\partial \phi_{2}}\right) \\
& +2 k_{1} A\left(\lambda_{1} \frac{\partial^{3} \lambda_{1}}{\partial \phi_{1}^{3}}+\lambda_{2} \frac{\partial^{3} \lambda_{1}}{\partial \phi_{1}^{2} \partial \phi_{2}}+\lambda_{2} \frac{\partial^{3} \lambda_{2}}{\partial \phi_{1} \partial \phi_{2}^{2}}\right) \\
& \left.+2 k_{2} A\left(\lambda_{2} \frac{\partial^{3} \lambda_{2}}{\partial \phi_{2}^{3}}+\lambda_{1} \frac{\partial^{3} \lambda_{2}}{\partial \phi_{1} \partial \phi_{2}^{2}}+\lambda_{2} \frac{\partial^{3} \lambda_{1}}{\partial \phi_{1} \partial \phi_{2}^{2}}\right)\right] d \phi_{1} \wedge d \phi_{2} \wedge d \theta \\
& +k_{\zeta}^{\perp} .
\end{aligned}
$$

\section{Summary}

We showed that the moduli space of the near horizon metric of five dimensional extremal black holes that are asymptotically flat or $\operatorname{AdS}$ has an $\mathrm{SL}(2, \mathbb{Z})$ modular symmetry which

\footnotetext{
${ }^{4}$ Our convention is $\epsilon_{\operatorname{tr} \phi_{1} \phi_{2} \theta}=1$.
} 
leaves the Bekenstein-Hawking entropy invariant. By using the Brown-Henneaux approach, we obtained CFTs associated to the points in moduli space which are identified by the modular group. In this way we realized a lattice of chiral CFTs dual to any such geometry. The microscopic entropy of each lattice point is equivalent to the Bekenstein-Hawking entropy.

\section{Acknowledgement}

We would like to thank R. Fareghbal for valuable discussions.

\section{References}

[1] A. Strominger and C. Vafa, Microscopic Origin of the Bekenstein-Hawking Entropy, Phys. Lett. B379 (1996) 99104, hep-th/9601029.

[2] M. Guica, T. Hartman, W. Song and A. Strominger, The Kerr/CFT correspondence, arXiv:0809.4266.

[3] J. D. Brown and M. Henneaux, Central Charges in the Canonical Realization of Asymptotic Symmetries: An Example from Three-Dimensional Gravity, Commun. Math. Phys. 104 (1986) 207226.

[4] A. Strominger, Black Hole Entropy from Near-Horizon Microstates, JHEP 02 (1998) 009, hep-th/9712251.

[5] S. Carlip, Black hole entropy from conformal field theory in any dimension, Phys. Rev. Lett. 82 (1999) 2828, hep-th/9812013.

[6] S.N. Solodukhin, Conformal description of horizons states, Phys. Lett. B454 (1999) 213, hep-th/9812056.

[7] M.I. Park, Hamiltonian dynamics of bounded spacetime and black hole entropy: Canonical method, Nucl. Phys. B634 (2002) 339, [hep-th/0111224].

[8] H. Lü, J. Mei and C.N. Pope, Kerr-AdS/CFT Correspondence in Diverse Dimensions, arXiv:0811.2225.

[9] H. Isono, T.S. Tai and W.Y. Wen, Kerr/CFT correspondence and five-dimensional $B M P V$ black holes, arXiv:0812.4440.

[10] T. Azeyanagi, N. Ogawa and S. Terashima, Holographic Duals of Kaluza-Klein Black Holes, arXiv:0811.4177.

[11] David D.K. Chow, M. Cvetic, H. Lü and C.N. Pope, Extremal Black Hole/CFT Correspondence in (Gauged) Supergravities, arXiv:0812.2918.

[12] Y. Nakayama, Emerging AdS from Extremaly Rotating NS5-branes, arXiv:0812.2234.

[13] T. Azeyanagi, N. Ogawa and S. Terashima, The Kerr/CFT Correspondence and String Theory, [arXix:0812.4883]. 
[14] H. Kunduri, J. Lucietti and H.S. Reall, Near-horizon symmetries of extremal black holes, Class. Quant. Grav.24 4269-4190, 2007 arXiv:0705.4214].

[15] G. Barnich and F. Brandt, Covariant theory of asymptotic symmetries, conservation laws and central charges, Nucl. Phys. B633 (2002) 382, [hep-th/0111246].

[16] G. Barnich and G. Compere, Surface charge algebra in gauge theories and thermodynamic integrability, J. Math. Phys. 49 (2008) 042901, arXiv:0708.2378.

[17] V.P. Frolov and K.S. Thorne, Renormalized stress-energy tensor near the horizon of a slowly evolving, rotating black hole, Phys. Rev. D39 (1989) 2125.

[18] T. Hartman, K. Murata, T. Nishioka, and A. Strominger, CFT Duals for extreme Black Holes, arXiv:0811.4393.

[19] F. Loran, H. Soltanpanahi, Near the horizon of 5D black rings, arXiv:0810.2620. 